\title{
Role of Anterior Approach in Management of Bilateral Cervical Locked Facet
}

\author{
RAMI TEAMA, M.D. \\ The Department of Neurosurgery, Faculty of Medicine, Benha University, Egypt
}

\begin{abstract}
Background: Cervical facet dislocation is the forward displacement of one cervical vertebra relative to another.

Aim of Study: The aims of surgical intervention are realignment and, decompression of the compressed neural structures and instrumentation to stabilize the injured spine. Anterior approaches can regain normal cervical curvature and associated with little postoperative pain, better wound healing and appearance and less wound infection.
\end{abstract}

Patients and Methods: A prospective study of 19 patients with bilateral cervical locked facet who underwent surgical treatment at benha university hospitals over a period of three years (Jan. 2017 / December 2019) is presented. Carefull general and neurological examination was done with preoperative CT cervical spine as the main investigation. All cases were operated using anterior approach with the use of casper screws reduction technique to restore and stabilize the cervical curve.

Results: The study group contained 16 women and 3 men. The age of patients in this study ranged from 21 years to 54 years and the mean age was 37 years. The mean follow-up period was 18 months. 15 cases presented with incomplete spinal cord injury. There were no intraoperative mortality or surgery related complications. 3 cases showed improvement while 2 cases died during follow-up due to chest problems.

Conclusion: Anterior cervical decompression, reduction using casper pins elevation technique and fusion using intervertebral cage and plate system is very safe, effective method for the management of bilateral subaxial cervical locked facet with great success rate and very few complications in addition to familiarity to most neurosurgeons.

Key Words: Cervical-Locked facet-Anterior approach.

\section{Introduction}

CERVICAL facet dislocation is the forward displacement of one cervical vertebra relative to another [8]. Hyperflexion is the most common mechanism of injury encountered in patients with such type of trauma, the injury may be caused by various mechanisms such as compressive hyper-

Correspondence to: Dr. Rami Teama,

E-Mail: ramyteama82@gmail.com flexion, vertical compression and distractive hyperflexion [11].

The aims of surgical intervention are realignment and regain of normal cervical balance, decompression of the compressed neural structures and instrumentation to stabilize the injured spine using a solid implant [9]. Surgical treatment is well accepted for patients with traumatic cervical facet joint dislocations (CFD), but which approach is better is still a point of difference between surgeons: anterior, posterior or combined. Anterior approaches can regain normal cervical curvature and associated with little postoperative pain, better wound healing and appearance and less wound infection. Posterior approaches allow direct access to the locked facet joints. Combined anterior posterior approaches may be of value in more complex situations [5]

In this study I will review my experience in anterior decompression and fixation for management of cervical subluxation associated with bilateral locked facet using screw reduction technique.

\section{Patients and Methods}

This is a prospective study including 19 operated cases by me as the main surgeon. These cases came to our Neurosurgery Department at Benha University and were diagnosed as posttraumatic single level bilateral cervical locked facet in the period from January 2017 to December 2019. The cause of trauma was road traffic accidents (RTA) in most cases (15), fall from a height (3) and diving injury in one patient.

All patients subjected to careful general examination with special attention given to vital signs and other system injury. All patients subjected to plain X-ray cervical spine lateral and AP views, CT cervical spine with sagittal and coronal 3D reconstruction, and MRI cervical spine. 
The most suitable investigation was CT cervical spine with sagittal and coronal 3D reconstruction. we followed-up patients by palin X-ray cervical spine either alone or in association with CT cervical spine in some cases one day postoperative, 3 months postoperative, 6 months postoperative and then at yearly interval.

\section{Surgical technique:}

- General anesthesia in supine position with neck collar applied in all cases.

- Shoulder traction by adhesive tape and a roll was placed in the upper iterscapular area and head supported by roll then the neck collar was removed.

- Sterilization of the operative field using betadine and alcohol.

- Right sided anterior cervical approach was used in all cases (Smith-Robinson approach).

- Microsurgical cervical discectomy of the affected level.
- Incisioning of the posterior longitudinal ligament.

- Reduction was performed using Ordonez, et al., technique (Fig. 1):

- Application of Casper retractor with 10 to 20 degree divergent angle.

- Reduction was done by gradual careful opening of the Casper with application of gentle backward pressure on the upper vertebrae and manual gentle neck traction under fluoroscopy till reduction occurs, then we closed the Casper forceps to a degree that allow the facets to return to its normal position.

- Intervertebral cage placement followed by removal of Casper forceps.

- Fixation using anterior suitable length plate.

- Palin radiographs was done in AP and lateral views using $\mathrm{C}$ arm.

- Closure of wound over non suction drain.

- Philadelphia neck collar was used in all patients for at least two months.

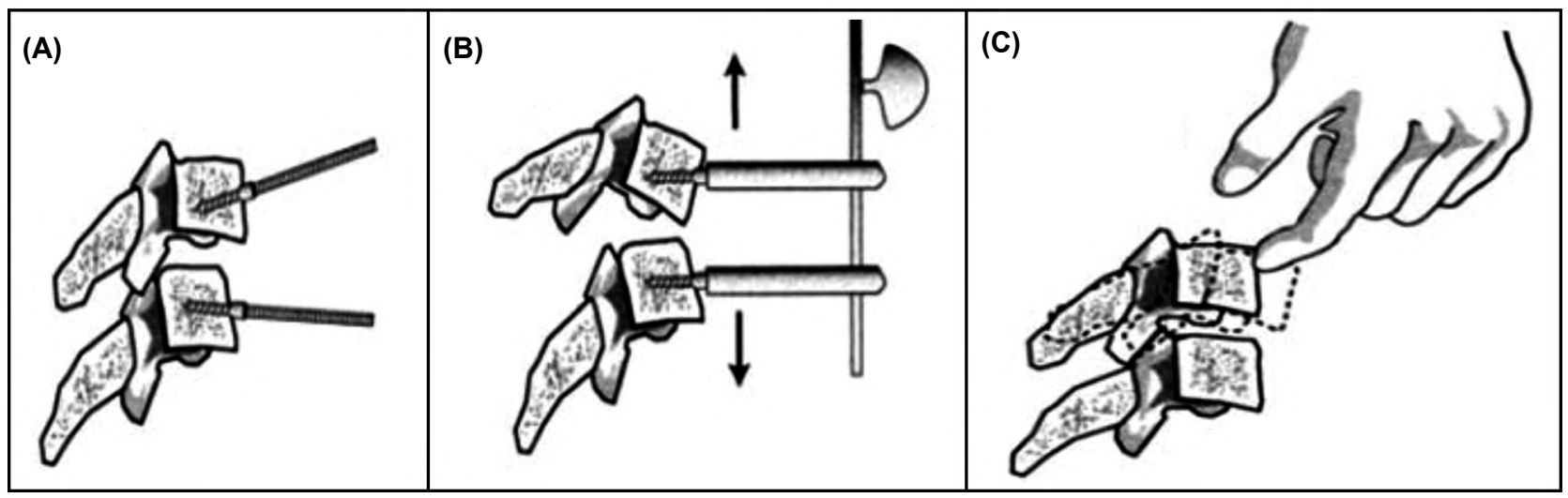

Fig. (1A-C): A: Placing distractor pins. B: Distraction disengages the facets. C: Dorsal force applied by manual pressure permits reduction of the dislocation [10]

\section{Results}

\section{Patient data:}

The study group was contained 16 women and $3 \mathrm{men}$. The age of patients in this study ranged from 21 years to 54 years and the mean age was 37 years. The mean follow-up period was 18 months. In this study 15 patients presented with incomplete spinal cord injury (ICI), 3 cases presented with complete spinal cord injury (CSCI) and 1 case with root symptoms. The most affected level was C 6-7 in 10 patients, C 5-6 in 6 cases, C4-5 in 3 cases.
Table (1): Patient criteria.

\begin{tabular}{lll}
\hline Criteria & Number of cases & $\%$ \\
\hline Sex: & & \\
F & 16 & 84.2 \\
M & 3 & 15.8 \\
Mean age & 37 & \\
Affected level & - C6-7/10 & 52.6 \\
& - C5-6/6 & 31.5 \\
Clinical presentation & - C4-5/3 & 15.8 \\
& - ICI/15 & 78.9 \\
& - CSCI/3 & 15.78 \\
Cause of trauma & - Root manif/1 & 5.26 \\
& - RTA/ 15 & 79 \\
& - Fall from height/3 & 15.8 \\
& - Diving/1 & 5.3 \\
\hline
\end{tabular}




\section{Perioperative management:}

All cases except the 3 cases with complete cord injury were subjected to high dose methylprednisolone therapy. Closed reduction was tried in most cases but unfortunately no reduction was achieved in most cases, so in the later cases no closed reduction was tried. The three cases with complete spinal cord injury were admitted to ICU but no ventilator was used while the rest of cases needed no ICU admission. Surgery was performed as early as possible in all cases (within 48 hours). Anterior approach was used for decompression and fixation and was successful in all cases. Philadelphia neck collar was used in all cases for at least two months. Physiotherapy was started according to general and neurological status of the patient and ability of the patient to tolerate pain.

\section{Intraoperative complications:}

There was no reported intraoperative morbidity or mortality.

\section{Postoperative outcome:}

Dysphagia was present in 3 cases and was improved using conservative management. Reduction was achieved radiologicaly in all cases.

Regarding the clinical outcome the three cases with complete spinal cord injury remained as such and rehabilitation was used in them, 6 months after surgery one case died from chest problems. Regarding cases with incomplete spinal cord injury, physiotherapy was used in them all, one patient died due to exaggerated diaphragmatic paralysis that was present in the preoperative stage, two cases with central cord syndrome showed improvement in neurological functions especially in lower limbs with regaining the ability to walk while the remaining cases kept the same perioperative neurological status. The one case with root symptoms showed complete improvement.

Table (2): Clinical outcome.

\begin{tabular}{lcc}
\hline Item & Number of cases & $\%$ \\
\hline Improvement & 3 & 15.78 \\
The same status & 14 & 73.68 \\
Deterioration & 2 & 10.52 \\
\hline
\end{tabular}

\section{Illustrative cases:}

Case (1):

Fifty four years old female presented with complete spinal cord injury. The affected level was C 6/7. Closed Reduction was tried but failed. Surgery was performed the day after admission using anterior decompression and fixation. Rehabilitation started after discharge (Fig. 2).

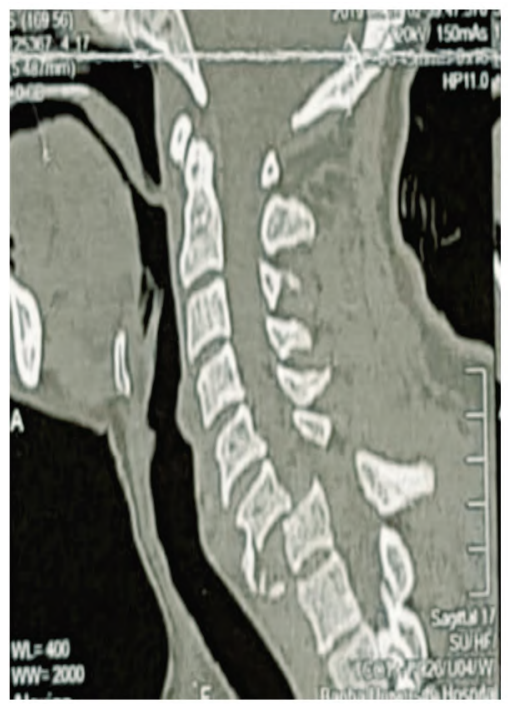

(A)

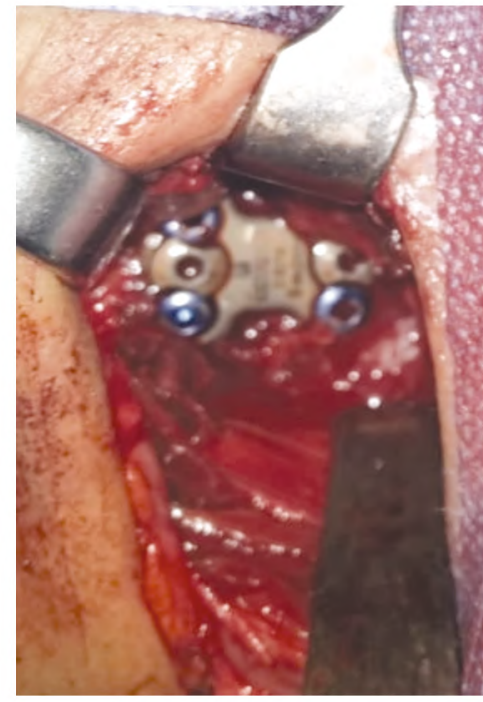

(B)

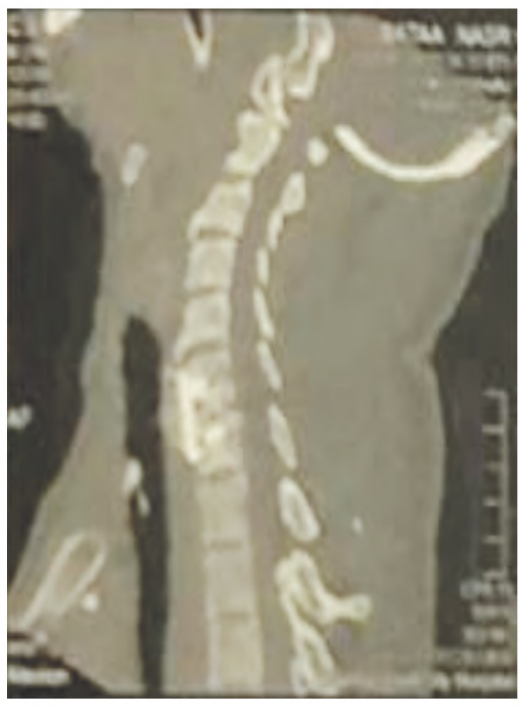

(C)

Fig. (2): A: Preoperative CT cervical spine with sagittal reconstruction showing complete dislocation at the level of C6/7. B: intraoperative view following reduction and stabilization using intervertebral cage and plate. C: Postoperative CT cervical spine with sagittal reconstruction done 1 day postoperative showing complete reduction with good alignment.

Case (6):

Thirty six old female presented with quadriparesis. Investigations revealed subluxation at C6/7 in addition to type II odontoid fracture. Surgery was done within 10 hours of admission. Ventral decompression and fixation was done and odontoid compression screw was inserted as well. Physical therapy was started after surgery. The case showed good results after surgery with residual mild lower limb weakness (grade IV power) and return to her usual activities (Fig. 3). 

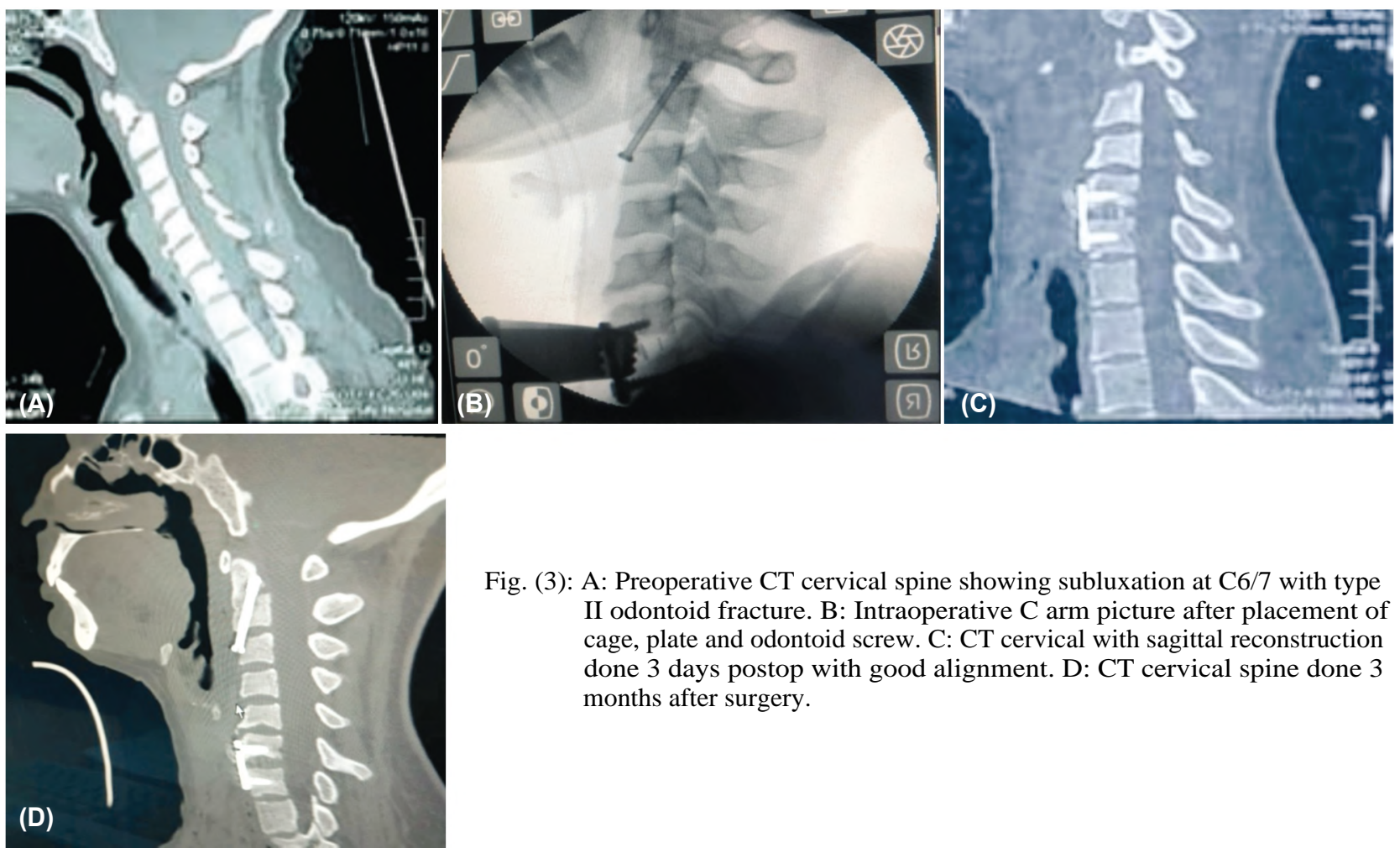

Fig. (3): A: Preoperative CT cervical spine showing subluxation at C6/7 with type II odontoid fracture. B: Intraoperative $\mathrm{C}$ arm picture after placement of cage, plate and odontoid screw. C: CT cervical with sagittal reconstruction done 3 days postop with good alignment. D: CT cervical spine done 3 months after surgery.

Case (11):

Forty years old female presented with central cord syndrome. Investigations revealed bilateral cervical facet dislocation with subluxation at level of C5/6. Surgery was done as early as pos- sible within 24 hours using anterior approach for decompression and fixation with good radiological outcome but no improvement occurs (remained quadriparetic) despite intense physiotherapy (Fig. 4).

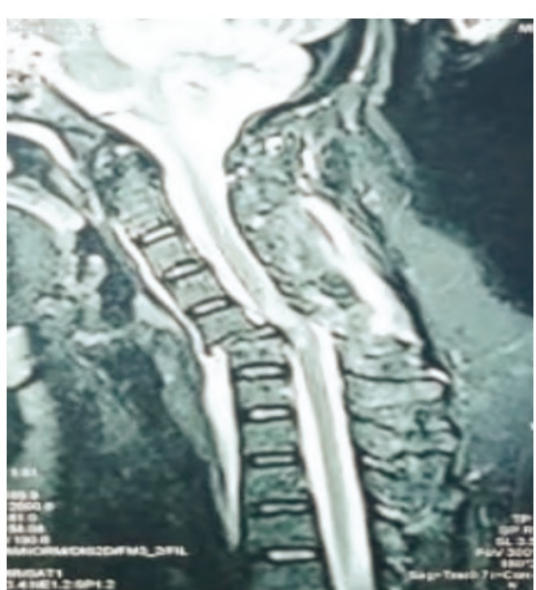

(A)

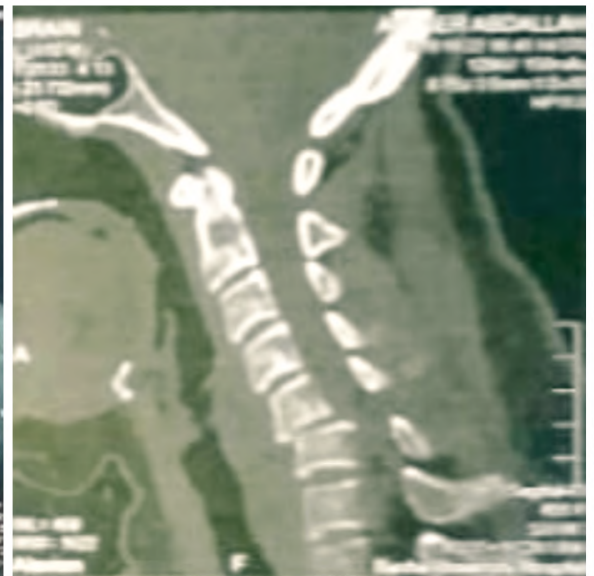

(B)

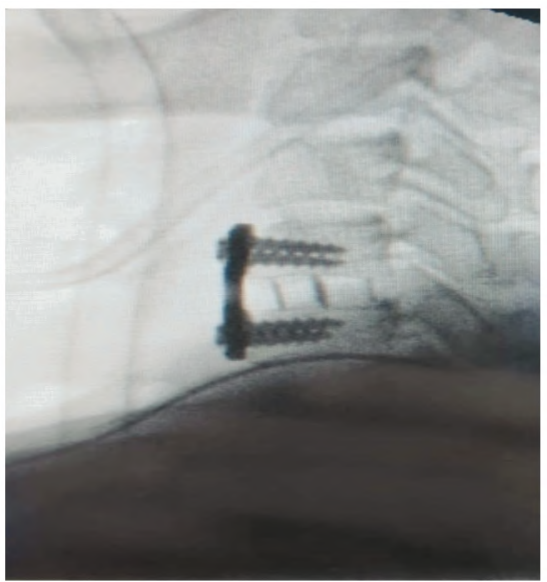

(C)

Fig. (4): A: Preoperative sagittal MRI showing subluxation at C5/6 with cord hematoma opposite that level. B: Preoperative CT with sagittal reconstruction. C: Early postoperative X-ray while the patient was in ICU showing complete reduction with good alignment.

\section{Discussion}

In a study performed by liu \& zhang including Sixty-three patients with unilateral/bilateral facet dislocation of the subaxial cervical spine. The authors used anterior approach for decompression and fixation. In 52 patients successful reduction was performed using our technique (distraction with Caspar pins). In the rest of cases that show failure with the former technique the authors used the usual technique of anterior facetectomy technique. The authors did not use posterior approach. Improvement happened in 23 patients after this procedure, and no neurologic deterioration hap- 
pened in any case. After at least 12 months of follow-up, good radiological fusion was observed in all cases, no construct failure was observed [6] Liu performed another study to compare the anterior approach with combined anterior posterior approach and the conclusion was that the anterior approach alone leaded to complete fusion in all cases in addition to reduction of surgical trauma so anterior approach alone is enough for such cases [7].

In a study performed by Kim et al., including Twenty-three patients with single level cervical facet fracture dislocation in whom the anterior approach alone was used following intraoperative closed reduction under general anesthesia. Anterior decompression and fixation was succefull in all cases except for two cases that showed failure of closed reduction and open anterior reduction due to risk of overdistraction. In these two cases the authors performed posterior facetectomy first followed by anterior discectomy and fixation. There was only one complication in the form of screw retropulsion that occurred 1 month following surgery. No deterioration in neurological status occurred. Stability of fusion was observed in all cases during final follow-up [3].

In another study performed by Ordonez et al., including six patients presented with unilateral cervical facet dislocation and four patients with bilateral cervical facet dislocation. All patients underwent anterior decompression, reduction of the dislocation, and fusion of the cervical spine by the same way I used. The technique was succeful in all cases except one patient that showed failure of anterior approach. The authors used posterior facetectomy and lateral mass fixation followed by anterior cervical discectomy and plate fixation. No intraoperative complications occurred. Improvement occurred in six patients and the remaining four remain as such. No neurological deterioration was observed during follow-up [10] .

Another series that support the effectiveness of anterior approach was done by Miao et al., They recommended that alone anterior approach is sufficient for reduction, decompression and fixation of subaxial cervical facet dislocation even in the most severe form. This series included forty cases with severe lower cervical facet dislocation that were managed by closed reduction followed by anterior fixation alone with no reported failure in all cases. In this study neurological functions improved in all cases with good bony fusion during the first six months after surgery [8].

Lins et al., after performing a study in the literature to compare various approaches used for management of cervical facet dislocation concluded that there is no approach to be superior to another and that the surgeon should be able to use both approaches to manage such cases [5].

Kanna et al., performed another study to support the use of anterior approach for management of subaxial cervical facet dislocation. The advantages of this approach were the same as those in our study and include, succeful reduction, familiarity of approach, minimal blood loss, lower infection rate, short good fusion. This study included 39 patients with both bilateral and unilateral locked facet [2].

In this series I used the technique of anterior cervical distraction using screws of casper pins and it was safe and effective technique. Li et al., performed a study to comparethe same technique and conventional anterior cervical reduction technique for traumatic cervical spine fractures and dislocations and said that Anterior cervical distraction and screw elevating-pulling reduction is simple with low risk, shortened operative time, good radiological and clinical outcome. Another conclusion was the great safety of the procedure in form of reduction of postoperative complications and of the iatrogenic cervical spinal cord injury.

This study included two groups, group A (44 cases operated using conventional anterior cervical reduction) and group B (42 cases of anterior cervical distraction and screw elevating-pulling reduction) [4]

Ahmed et al., performed a study to show the effectiveness of closed reduction in management of subaxial cervical spine dislocation and the failure rate was also high as that in our study. They Saied that delayed admission was the cause and recommended early admission and closed reduction in the emergency room.

\section{Conclusion:}

Anterior cervical decompression, reduction using casper pins elevation technique and fusion using intervertebral cage and plate system is very safe, effective method for the management of bilateral subaxial cervical locked facet with great success rate and very few complications in addition to familiarity to most neurosurgeons.

\section{References}

1- AHMED W.A., NAIDOO A. and BELCI M.: Rapid incremental closed traction reduction of cervical facet fracture dislocation: The Stoke Mandeville experience. Spinal Cord Ser Cases, 4: 86, 2018. 
2- KANNA R.M., SHETTY A.P. and RAJASEKARAN S.: Modified anterior-only reduction and fixation for traumatic cervical facet dislocation. Eur. Spine Journal, 27 (6): 1447-1453, 2018.

3- KIM S.G., PARK S.J., WANG H.S., JU C.I., LEE S.M. and KIM S.W.: Anterior Approach Following Intraoperative Reduction for Cervical Facet Fracture and Dislocation. Korean Neurosurg Soc., 63 (2): 202-209, 2020.

4- LI H., HUANG Y., CHENG C. and LIN Z.: Comparison of the technique of anterior cervical distraction and screw elevating-pulling reduction and conventional anterior cervical reduction technique for traumatic cervical spine fractures and dislocations. International Journal of Surgery, 40: 45-51, 2017.

5- LINS C.C., PRADO D. and JOAQUIM A.: Surgical treatment of traumatic cervical facet dislocation: Anterior, posterior or combined approaches?. Arq. neuro-psiquiatr, 47, 2016.

6- LIU K. and ZHANG Z.: A Novel Anterior-Only Surgical Approach for Reduction and Fixation of Cervical Facet Dislocation. World Neurosurgery, 128: 362-369, 2019.
7- LIU K. and ZHANG Z.: Comparison of a novel anterioronly approach and the conventional posterior-anterior approach for cervical facet dislocation: A retrospective study. Eur. Spine J., 28 (10): 2380-2389, 2019.

8- MIAO D.C., QI C., WANG F., LU K. and SHEN Y. Management of Severe Lower Cervical Facet Dislocation without Vertebral Body Fracture Using Skull Traction and an Anterior Approach. Med. Sci. Monit., 24: 12951302, 2018.

9-OLVERA D.Q., OLVERA J.Q., RESENDIZ J.Q. and GARCIA F.V.: Surgical treatment for cervical subluxation caused by trauma: A report case and literature review. Trauma and Emergency Care, 4: 1-4, 2019.

10- ORDONEZ B.J., BENZEL E.C., NADERI S. and WELLER S.J.: Cervical facet dislocation: Techniques for ventral reduction and stabilization. J. Neurosurg., 92 (1 Suppl): 18-23, 2000.

11-RANIGA S.B., MENON V., KHAMIS S., AL MUZAHMI and BUTT S.: (2014) MDCTof acute subaxial cervical spine trauma: A mechanism-based approach, Insights Imaging, 5: 321-338.

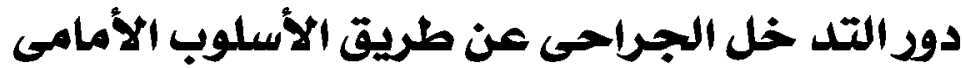

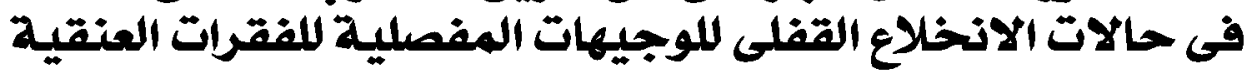

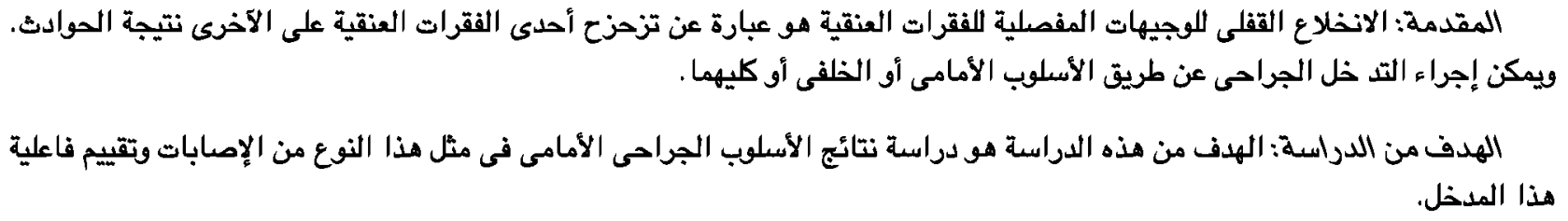

